\title{
Neutron Star Model Atmospheres
}

\author{
Valery Suleimanov*, Thomas Rauch*, Klaus Werner*, Alexander Y. \\ Potekhin $^{\dagger}$ and Juri Poutanen** \\ ${ }^{*}$ Institute for Astronomy and Astrophysics, Kepler Center for Astro and Particle Physics, \\ University of Tübingen, 72076 Tübingen, Germany \\ ${ }^{\dagger}$ Ioffe Physical-Technical Institute, Polytekhnicheskaya str. 26, St. Petersburg 194021, Russia \\ ${ }^{* *}$ Astronomy Division, Department of Physical Sciences, 90014 University of Oulu, Finland
}

\begin{abstract}
Determination of neutron star (NS) parameters is an important astrophysical problem. The stellar radius depends on the mass and the equation of state (EOS) in the NS core, which is not well known. Therefore, with masses and radii inferred from observations we are able to select between various theories for the EOS. Theoretical models of NS spectra are needed for this purpose.
\end{abstract}

Keywords: Equations of state of neutron-star matter - Neutron stars - Stellar atmospheres Accretion and accretion disks

PACS: $26.60 . \mathrm{Kp}, 97.60 . \mathrm{Jd}, 97.10 . \mathrm{Ex}, 97.10 . \mathrm{Gz}$

Absorption features in spectra of X-ray bursting NSs. Some low mass X-ray binaries (LMXBs) with relative low mass-accretion rate are $\mathrm{X}$-ray bursting sources due to eruptive $\mathrm{H} / \mathrm{He}$ thermonuclear burning of accreted matter on the NS surface. The luminosity during outburst maximum can reach the Eddington limit $\left(T_{\text {eff }} \sim 2.5 \times 10^{7} \mathrm{~K}\right)$ and then decrease exponentially on a time scale of a few seconds. Cottam et al. [1] found an absorption feature at 13-14 $\AA$ in EXO 0748-676 during late phases of an outburst, identified it as an Fe XXV subordinate line and estimated the gravitational redshift on the NS surface $(z \approx 0.35)$. We checked this identification using NS model atmospheres.

Compton scattering determines atmospheric structures and emergent spectra at outburst maximum, but it is less important at $T_{\mathrm{eff}} \leq 1.5 \times 10^{7} \mathrm{~K}$ (for atmospheres with solar chemical composition). We calculated a set of non-LTE and LTE models with various chemical abundances [5] and demonstrated that the observed absorption could rather be a blend of Fe XXIV resonance lines with gravitational redshift $z \approx 0.24$. But the equivalent width of this blend is too low in comparison to the observation, therefore this feature can probably be not connected with surface radiation. In subsequent outburst observations, no absorption at this place in the spectrum was found [2].

Spectra of spreading layers and EOS in NS cores. X-ray spectra of high luminosity LMXBs consist of two blackbody components with $k T \sim 1 \mathrm{keV}$ and $\sim 2.5 \mathrm{keV}$. The more variable hard component is associated with the boundary layer (BL) between accretion disc and NS surface. Inogamov \& Sunyaev [4] suggested a new, so-called spreading layer (SL) model for the BL. In this model accreted matter spirals with subkeplerian velocity, spreads over the NS surface, and decelerates due to friction with the surface material. We developed an SL model by accounting for General Relativity (GR) corrections and considering an arbitrary chemical composition of the accreting matter. We also computed emergent spectra taking into account all GR corrections. 
We compared them to observations and obtained limits on the NS $M-R$ relation and, therefore, on the EOS in NS cores.

For computing the total spectrum we divided the SL model in a number of latitude rings with known $T_{\text {eff }}$, effective surface gravity, surface density, and calculated their vertical structures and spectra by using a model atmosphere method with Compton scattering taken into account. The local vertical structures and the local spectra are very similar to X-ray bursting NS atmospheres. We concluded, for example, on a radius of $R=14.5 \pm 1.5 \mathrm{~km}$ for a NS with $M=1.4 \mathrm{M}_{\odot}$, which is evidence for a stiff EOS in NS cores [6].

Atmospheres of magnetized neutron stars. In the last two decades, several new classes of isolated NSs with strong magnetic fields $B \sim 10^{13}-10^{14} \mathrm{G}$ have been discovered: dim isolated NSs, anomalous X-ray pulsars, and soft-gamma repeaters. Their spectra are close to black body spectra with $T \sim 10^{6}-10^{7} \mathrm{~K}$. They can have gaseous envelopes, therefore, model atmospheres of magnetized NSs have to be calculated.

A magnetized plasma is birefringent, thus, electromagnetic waves have to be considered in two normal modes, the extraordinary (X-mode), elliptically polarized across magnetic field direction, and the ordinary (O-mode), elliptically polarized along magnetic field direction. The X-mode opacity is smaller by a factor of $\sim\left(v / v_{\text {cyc }}\right)^{2}$, where $v_{\text {cyc }}$ is the electron-cyclotron frequency. Opacities in both modes are strongly dependent on the angle between a magnetic field direction and the wave propagation direction. In fields with a strength near $B_{\mathrm{Q}}=4.4 \times 10^{13} \mathrm{G}$, virtual $e^{+} e^{-}$pairs modify the plasma properties and opacities. For a given $v$ and $B$ there is some critical plasma density where the $\mathrm{X}$-mode opacity sharply increases (vacuum resonance) and the radiation modes can partially convert into each other. Atomic binding energies are increased in strong magnetic fields, and even $\mathrm{H}$ can be only partially ionized at $T \sim 10^{6} \mathrm{~K}$. Fully ionized $\mathrm{H} / \mathrm{He}$ model atmospheres of magnetized NSs were calculated by many authors, including vacuum polarization and partial mode conversion [8]. Partially ionized $\mathrm{H}$ models were also calculated [3], but without an accurate treatment of partial mode conversion.

We presented a new computer code for magnetized NS atmosphere modeling. The atmosphere is assumed to consist either of fully ionized electron-ion plasmas or of partially ionized hydrogen. Vacuum resonance and partial mode conversion are taken into account. Any inclination of the magnetic field relative to the stellar surface is allowed. Some new results were obtained, in particular the effect of the vacuum polarization on partially ionized hydrogen model atmospheres was investigated [7].

\section{REFERENCES}

1. Cottam, J., Paerels, F., \& Méndez, M. 2002, Nature, 420, 51

2. Cottam, J., Paerels, F., Méndez, M., et al. 2008, ApJ, 672, 504

3. Ho, W. C. G., Potekhin, A. Y., \& Chabrier, G. 2008, ApJS, 178, 102

4. Inogamov, N. A., \& Sunyaev, R. A. 1999, Astr. Letters, 25, 269

5. Rauch, T., Suleimanov, V., \& Werner, K. 2008, A\&A, 490, 1127

6. Suleimanov, V., \& Poutanen, J. 2006, MNRAS, 369, 2036

7. Suleimanov, V., Potekhin, A. Y., \& Werner, K. 2009, A\&A, in press (arXiv:0905.3276)

8. van Adelsberg, M, \& Lai, D. 2006, MNRAS, 373, 1495 